CHAPERONES

\section{Better with BACON}

Cell 150, 987-1001 (2012)

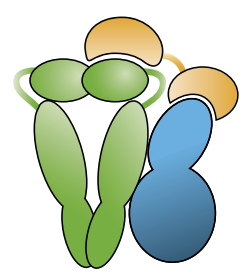

HSP90 is a chaperone that interacts with a diverse group of substrate proteins or clients, but the rules that govern client selection and HSP90-client interactions are unclear. Taipale et al. now report 'LUMIER with BACON', an assay involving a bait protein tagged with a Flag epitope and a prey protein tagged with Renilla luciferase. The proteins are coexpressed, and bait and prey are quantified by ELISA and luminescence, respectively, in high-throughput format after immunoprecipitation. Using their assay, the authors evaluated several client classes for interaction with HSP90 and found that $\sim 60 \%$ of human kinases interacted with HSP90. The authors next showed that the majority of kinases but not other client types also interacted with the cochaperone CDC37, suggesting that CDC37 mediates kinase-HSP90 interaction. Chemical inhibition of HSP90 with a diverse set of inhibitors generally decreased HSP90kinase interactions but with client-specific dissociation rates. Analysis of functional and structural databases suggested that HSP90 clients might be less stable than nonclients.
The authors showed that stabilization of the kinase domain of clients with small molecules that bind the ATP-binding pocket decreased the kinase-HSP90 interaction. Taken together, the data indicate that the CDC37 cochaperone is an adaptor for protein kinases and that, within this class of proteins, HSP90 clients are intrinsically unstable compared to nonclients.

\section{NEUROSCIENCE}

\section{Fixing fragility}

Sci. Transl. Med., published online 19 September 2012; doi:10.1126/scitranslmed.3004218

Sci. Transl. Med., published online 19 September 2012; doi:10.1126/scitranslmed.3004214

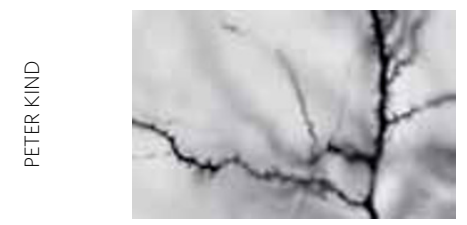

Fragile X syndrome (FXS) is caused by expansion of a CGG triplet repeat in the FMR1 gene (gene product FMRP), leading to transcriptional silencing of FMR 1 and deficiency of FMRP, which results in deregulated synaptic protein synthesis, deficiencies in GABA-mediated inhibitory neurotransmission and exaggerated responses to metabotropic glutamate receptors (mGluRs) that underlie cognitive and behavioral deficits in FXS patients. As GABA receptors inhibit glutamate release, Henderson et al. tested whether GABA receptor activation could indirectly reduce postsynaptic mGluR

\section{SYNTHETIC BIOLOGY}

Genetic room to breathe
Nat. Biotechnol., published online

16 September 2012; doi:10.1038/nbt.2355

Synthetic biology efforts have generated modular gene circuits that enable the engineering of genetic and metabolic pathways that are integrated with or are orthogonal to normal cellular pathways. However, experience has shown that the complex regulatory elements that govern transcription and translation can confound synthetic biology efforts that require precise, switchable and predictable changes in gene expression within cells. Qi et al. report a way to mitigate these challenges by fragmenting the mRNA of multigene constructs into modular functional expression units. The clustered regularly interspaced short palindromic repeat (CRISPR) pathway from bacteria-in which a CRISPR-associated endoribonuclease (Cys4) binds and cleaves at specific repetitive RNA sequences-was used as a targeted mRNA cleavage platform. By inserting CRISPR cleavage sites between promoters, 5' UTRs possibly containing regulatory sequences and open reading frames, the authors showed that CRISPR-mediated RNA processing could normalize previously variable protein expression across multiple genes of a construct, thereby rendering their composite behavior more predictable. Physical separation of RNA expression elements was also shown to enhance the responsiveness of individual modules to engineered regulatory motifs, such as antisense RNA. The demonstration that the system can be applied in several bacteria and in yeast suggests that CRISPR-mediated RNA processing may offer a useful adjunct tool to afford synthetic biologists greater control over their engineered circuits within cells.

activation and ameliorate the physiological and behavioral defects in the Fmrl knockout mouse model. They found that the $\mathrm{GABA}_{B}$ receptor agonist STX209 reduced synaptic protein synthesis and other molecular defects from Fmr1 deficiency, perhaps by affecting translation of the FMRP targets. STX209 also reduced seizure incidence and repetitive behavior in the mice and restored normal spine density of pyramidal neurons from the binocular visual cortex, a correction of the pruning and shaping defects of the neuronal network that underlie FXS. In a parallel study, Berry-Kravis et al. tested the effects of STX209 on one of the core phenotypes of human FXS, the social impairment that is thought to be due to exaggerated responses to mGluRs. In a post hoc analysis, the authors saw improvements in social avoidance, consistent with a model where social impairments in FXS are related to GABA deficiencies or exaggerated glutamatergic signaling. These studies add STX209 to the repertoire of potential FXS drug leads, such as agents that modulate mGluR receptors directly.

MOLECULAR BIOLOGY

\section{You can leave your cap on}

Nat. Struct. Mol. Biol., published online

9 September 2012; doi:10.1038/nsmb.2381

The $5^{\prime}$ end of mRNA in eukaryotes is modified-or 'capped' - with a methylated guanine nucleotide to protect the mRNA from impromptu degradation. Chang et al. report that the yeast enzyme Ydr370C (renamed Dxo1), which is weakly homologous to the recently discovered RNA pyrophosphohydrolase and decapping enzyme Rai1, may be responsible for detecting and degrading incompletely capped mRNAs that are mistakenly exported into the yeast cytoplasm. The authors solved the X-ray crystal structure of Dxo1 and assessed the biochemical properties of the two enzymes. Rail was previously shown to act as a pyrophosphohydrolase, removing a pyrophosphate group from uncapped RNA that had a triphosphate group on its $5^{\prime}$ end, and as a decapping enzyme, completely removing the $5^{\prime}$ cap of a capped, unmethylated RNA. Dxo1 was also able to remove the $5^{\prime}$ cap of a capped, unmethylated RNA, but it was not able to catalyze the pyrophosphohydrolase reaction. In addition, Dxo1 was then able to act as a $5^{\prime}-3^{\prime}$ exoribonuclease, degrading the uncapped piece of RNA. The authors showed that knocking out both Dxol and Rail in yeast led to an increase in incompletely $5^{\prime}$-capped mRNA under normal growth conditions. They believe that these two enzymes-Rail in the nucleus and Dxo1 in the cytoplasm- 